\title{
Multislice CT angiography in the selection of patients with ruptured intracranial aneurysms suitable for clipping or coiling
}

\author{
H. E. Westerlaan • J. Gravendeel • D. Fiore • \\ J. D. M. Metzemaekers • R. J. M. Groen • \\ J. J. A. Mooij • M. Oudkerk
}

Received: 2 April 2007 / Accepted: 12 July 2007 / Published online: 22 September 2007

(C) Springer-Verlag 2007

\begin{abstract}
Introduction We sought to establish whether CT angiography (CTA) can be applied to the planning and performance of clipping or coiling in ruptured intracranial aneurysms without recourse to intraarterial digital subtraction angiography (IA-DSA).

Methods Over the period April 2003 to January 2006 in all patients presenting with a subarachnoid haemorrhage CTA was performed primarily. If CTA demonstrated an aneurysm, coiling or clipping was undertaken. IA-DSA was limited to patients with negative or inconclusive CTA findings. We compared CTA images with findings at surgery or coiling in patients with positive CTA findings and in patients with negative and inconclusive findings in whom IA-DSA had been performed.

Results In this study, 224 consecutive patients (mean age 52.7 years, 135 women) were included. In 133 patients (59\%) CTA demonstrated an aneurysm, and CTA was followed directly by neurosurgical $(n=55)$ or endovascular treatment $(n=78)$. In 31 patients (14\%) CTA findings were categorized as inconclusive, and in 60 (27\%) CTA findings were negative. One patient received surgical treatment on the basis of false-positive CTA findings. In 17 patients in whom CTA findings were inconclusive, IA-DSA provided further diagnostic information required for correct patient
\end{abstract}

H. E. Westerlaan $(\bowtie) \cdot J$. Gravendeel $\cdot$ D. Fiore $\cdot$ M. Oudkerk Department of Radiology, University Medical Center Groningen, Post Box 30.001, Hanzeplein 1,

9700 RB Groningen, The Netherlands

e-mail: H.E.Westerlaan@rad.umcg.nl

J. D. M. Metzemaekers • R. J. M. Groen · J. J. A. Mooij

Department of Neurosurgery,

University Medical Center Groningen,

Post Box 30.001, Hanzeplein 1,

9700 RB Groningen, The Netherlands selection for any therapy. Five ruptured aneurysms in patients with a nonperimesencephalic SAH were negative on CTA, and four of these were also false-negative on IADSA. On a patient basis the positive predictive value, negative predictive value, sensitivity, specificity and accuracy of CTA for symptomatic aneurysms were $99 \%, 90 \%$, $96 \%, 98 \%$ and $96 \%$, respectively.

Conclusion CTA should be used as the first diagnostic modality in the selection of patients for surgical or endovascular treatment of ruptured intracranial aneurysms. If CTA renders inconclusive results, IA-DSA should be performed. With negative CTA results the complementary value of IA-DSA is marginal. IA-DSA is not needed in patients with negative CTA and classic perimesencephalic SAH. Repeat IA-DSA or CTA should still be performed in patients with a nonperimesencephalic SAH.

Keywords Subarachnoid haemorrhage · CTA · Intracranial · Aneurysm

\section{Introduction}

Subarachnoid haemorrhage (SAH) is caused by aneurysmal rupture in $70-85 \%$ of patients $[1,2]$. In a systematic review, Hop et al. found fatality rates ranging from $32 \%$ to $67 \%$ [3]. Furthermore, $10-20 \%$ of patients remained functionally dependent after SAH. Rapid diagnostic evaluation and treatment are crucial for the patient's outcome.

Intraarterial digital subtraction angiography (IA-DSA) has been the main technique for detecting and characterizing intracranial aneurysms and remains the gold standard. However, IA-DSA is invasive and time consuming, and carries a risk of neurological complications of $0.5-1.8 \%$ 
with permanent deficit in $0.09-0.5 \%$ [4-6]. Serious nonneurological complications, which occur in $0.6 \%$ of patients, include groin hematoma, peripheral thromboembolism, transient hypotension and arteriovenous fistulas [4]. Furthermore, IA-DSA may increase the risk of rebleeding $[7,8]$. It has been demonstrated that three-dimensional CT angiography (3D-CTA) can reliably detect intracranial aneurysms [9-14]. Only after replacement of IA-DSA by CTA can the advantages of CTA be fully realized in the clinical setting. We report here our clinical experience with both 16- and 64-detector row CTA as the first and intended only diagnostic and treatment decision-making study for intracranial aneurysms in patients with acute SAH.

\section{Materials and methods}

\section{Subjects}

Between April 2003 and January 2006 all patients presenting with a SAH to the University Medical Centre Groningen consecutively underwent CTA as the first diagnostic study. Based on the CTA findings, patients were selected for surgical clipping or endovascular coiling of a ruptured intracranial aneurysm.

SAH was suspected on clinical grounds and confirmed by unenhanced $\mathrm{CT}$ or by blood pigments on lumbar puncture.

\section{Imaging protocols}

The CT examinations were performed on a 16- or 64multidetector row spiral CT machine (Somatom Sensation 16 or 64; Siemens Medical Systems, Erlangen, Germany), based on a standard protocol. The 64-multisclice CT was implemented in the Emergency Department in December 2004.

- Parameters for 16-slice CT for diagnosis of aneurysm: via an intravenous cannula in the antecubital fossa, $80 \mathrm{ml}$ of contrast agent (Visipaque 320) was injected with a power injector at a rate of $4 \mathrm{ml} / \mathrm{s}$. Injection of contrast agent was followed by a flush of $50 \mathrm{ml}$ $0.9 \%$ saline (Stellant; $\mathrm{NaCl}$ Neck Angio) injected at the same rate. A manual fluoroscopic bolus-triggered system, with the internal carotid arteries as reference point and a delay of $4 \mathrm{~s}$, determined the optimal timing. The CTA protocol parameters were as follows: spiral mode, rotation time $0.5 \mathrm{~s}$, reconstruction interval $0.75 \mathrm{~mm}$ at Kernel $\mathrm{H} 20,120 \mathrm{kV} /$ $200 \mathrm{mAs}$, acquisition time $10 \mathrm{~s}$, scan range from the $\mathrm{C} 1$ vertebral body to the vertex parallel to the orbitomeatal line.

- Parameters for 16-slice CT for diagnosis of SAH: gantry un-angled, spiral mode, rotation time $0.75 \mathrm{~s}, 16-$ detector rows at $0.75-\mathrm{mm}$ intervals, table speed $6 \mathrm{~mm} /$ rotation, reconstruction interval $3 \mathrm{~mm}$ at Kernel H30 and acquisition parameters $120 \mathrm{kV} / 200 \mathrm{mAs}$. The actual acquisition time was approximately $15 \mathrm{~s}$.

- Parameters for 64-slice CTA for diagnosis of aneurysm: rotation time $1 \mathrm{~s}$, table speed $15.4 \mathrm{~mm} /$ rotation, reconstruction interval $0.6 \mathrm{~mm}$ at Kernel $\mathrm{H} 20$, $120 \mathrm{kV} / 260 \mathrm{mAs}$, acquisition time $9 \mathrm{~s}$ and scan range extending from the $\mathrm{C} 1$ vertebral body to the vertex parallel to the orbitomeatal line. The protocol parameters for contrast agent injection remained unchanged.

- Parameters for 64-slice CT for diagnosis of SAH: gantry un-angled, spiral mode, rotation time $1 \mathrm{~s}, 64$ detector rows at $0.6-\mathrm{mm}$ intervals, table speed $9.6 \mathrm{~mm} /$ rotation, reconstruction interval $2 \mathrm{~mm}$ at Kernel $\mathrm{H} 30$, acquisition parameters $120 \mathrm{kV} / 260 \mathrm{mAs}$ and acquisition time $14 \mathrm{~s}$.

\section{Postprocessing of CTA}

Source images were transferred to a remote computer workstation (Odelft Benelux diagnostic imaging) for viewing. Initial careful review of axial images was considered imperative. During this review any areas of concern could be noted. Two-dimensional maximum intensity projection (MIP) views and three-dimensional (3-D) surface-rendered and volume-rendered reconstructions were reformatted from the raw image date on a Vitrea computer workstation by one of the neuroradiologists.

\section{Parameters for IA-DSA and postprocessing}

From April 2003 until April 2004 the IA-DSA studies were produced on a digital angiographic unit (Siemens Multiskop with InfiMed image processing) with a $512 \times 512$ pixel matrix. From April 2004 onwards the studies were performed on a Siemens Axiom Artis angiographic unit with a $1024 \times 1024$ pixel matrix. Selective four- or six-vessel angiography using a standard projection format was performed initially and additional views were obtained if required to identify the parent vessel and aneurysm neck more clearly. The amount of contrast medium (Visipaque 270) used was $8 \mathrm{ml}$ for the internal carotid artery and $6 \mathrm{ml}$ for the external carotid artery, and the injection rate was $6 \mathrm{ml} / \mathrm{s}$ when the tip of the catheter was in the internal carotid artery and 3-4 ml/s when the tip of the catheter was in the external carotid artery. The rate of injection into the vertebrobasilar system was $6-8 \mathrm{ml} / \mathrm{s}$ to a total amount of $8 \mathrm{ml}$.

In certain situations, rotational 3-D angiography was performed to better delineate the anatomic details of an aneurysm. Rotational 3-D angiography was performed on a Siemens Axiom Artis angiographic unit. The $\mathrm{C}$-arm rotates in a continuous $200^{\circ}$ arc around the patient's head during a prolonged intraarterial catheter injection of contrast medi- 
um (28 $\mathrm{ml}$ Visipaque, injection rate $4 \mathrm{ml} / \mathrm{s})$. The raw date images were transferred to a Leonardo workstation (AX Applications) from which 3-D volume-rendered reconstructions were reformatted.

Image review and data analysis

The presence of an aneurysm, its size and morphology, its parent and feeding vessels and the collateral circulation at the circle of Willis were determined by one of the diagnostic or interventional neuroradiologists. If multiple aneurysms were detected, the usual criteria were applied to decide which aneurysm was responsible for the haemorrhage. These criteria included the unenhanced CT findings (distribution of blood) and the size and irregularity of the aneurysm.

All diagnostic findings were discussed with the neurosurgeons. The CTA results were categorized into proven ruptured aneurysm, inconclusive or negative. Patients with a proven ruptured aneurysm were selected subsequently for coiling or clipping. The surgical and endovascular findings were compared to the CTA findings. In general, ruptured aneurysms in the anterior circulation were selected for either coiling or clipping. Ruptured aneurysms located in the posterior circulation were preferably coiled. Giant intracranial aneurysms were preferably treated surgically. A ruptured aneurysm in association with an intraparenchymatous haemorrhage was most often selected for clipping of the aneurysm and surgical evacuation of the haematoma.

Patients categorized as inconclusive or negative underwent IA-DSA. In patients with a perimesencephalic blood distribution, one IA-DSA examination was performed. In patients with a nonperimesencephalic blood distribution a second IA-DSA was performed if the first one was negative. IA-DSA was considered the gold standard. CTA was considered false-negative when IA-DSA revealed a ruptured aneurysm or when rebleeding occurred.

The positive predictive value, negative predictive value, sensitivity, specificity and accuracy of CTA per patient were calculated. The chi-squared test was used to compare the performance of 16-slice CTA and 64-slice CTA for the identification of intracranial aneurysms. Differences with a $P$ value less than 0.05 were considered significant.

The IA-DSA findings in patients in the inconclusive category were compared with the CTA findings to assess whether IA-DSA actually provided any additional information.

\section{Results}

Patient population

From April 2003 until January 2006292 patients with SAH underwent CTA. Excluded from the study were 68 patients, of whom 24 were excluded because of a nonaneurysmal cause of the SAH including trauma $(n=17)$, arteriovenous malformation $(n=6)$ and anticoagulant therapy $(n=1), 3$ because of hypertension and intracerebral haematoma, 4 because of comorbidity or advanced age, 2 were excluded because of poor clinical condition and 1 because if poor clinical grading and advanced age, and 31 died from the initial effect of SAH, rebleed or vasospasm with ischemia. Two patients refused therapy and one patient was referred to another hospital for treatment.

The study included 224 patients, 89 men and 135 women with a mean $( \pm \mathrm{SD})$ age of $52.7 \pm 10.7$ years (range 22 79 years). Their clinical condition just before treatment was classified according to the original Hunt and Hess grading system: 99 patients were classified as grade I, 45 as grade II, 58 as grade III, 20 as grade IV, and 2 as grade V [15].

\section{Detection of intracranial aneurysms}

Of the 224 patients, 140 underwent 16-slice CTA and 84 underwent 64-slice CTA. The CTA results were categorized as proven ruptured intracranial aneurysm (133 patients, $59 \%$ ), inconclusive (31 patients, 14\%), or negative for aneurysm (60 patients, $27 \%$ ).

\section{Positive CTA result}

In this category 133 ruptured aneurysms were diagnosed in 133 patients. In 25 patients 32 associated unruptured aneurysms were diagnosed. The distributions of the locations and sizes of the aneurysms are shown in Tables 1 , 2, 3 and 4. An overview of the results in this subgroup is presented in Fig. 1.

The majority of patients were treated within 3 days of SAH $(n=99,75 \%)$. Of the 133 CTA-positive patients, 78 (59\%) were coiled and 55 (41\%) were clipped. Treatment conversion was needed in three patients, in two because of difficult aneurysm morphology and in one because of rebleeding during surgery (dura not yet opened). In two patients treatment conversion was necessary because of incorrect treatment selection based on CTA (Fig. 2).

All ruptured intracranial aneurysms were confirmed by surgery or endovascular treatment. In two patients IA-DSA was performed after surgical treatment for evaluation of coiling of asymptomatic aneurysms.

In four patients $(3 \%)$ a fatal rebleeding occurred during follow-up, in one patient soon after complete occlusion of the aneurysm with coiling, in one patient on the 5th day after clipping, in one patient 2 weeks after incomplete occlusion of the aneurysm with coiling and in one patient almost 3 years after clipping. In all patients the blood distribution of the rebleeding was the same as that of the primary SAH. Although permission was not granted for an 
Table 1 Location of symptomatic intracranial aneurysms in 224 patients

\begin{tabular}{|c|c|c|c|c|c|c|}
\hline \multirow[t]{2}{*}{ Aneurysm location } & & \multicolumn{2}{|c|}{$\begin{array}{l}\text { CTA-positive } \\
(n=133)\end{array}$} & \multicolumn{2}{|c|}{$\begin{array}{l}\text { CTA- } \\
\text { inconclusive } \\
(n=31)^{\mathrm{a}}\end{array}$} & \multirow[t]{2}{*}{$\begin{array}{l}\text { CTA- } \\
\text { negative } \\
(n=60)^{\mathrm{b}}\end{array}$} \\
\hline & & $\begin{array}{l}\text { Coiling } \\
(n=78)\end{array}$ & $\begin{array}{l}\text { Clipping } \\
(n=55)\end{array}$ & $\begin{array}{l}\text { Coiling } \\
(n=8)\end{array}$ & $\begin{array}{l}\text { Clipping } \\
(n=12)\end{array}$ & \\
\hline \multirow[t]{6}{*}{ Anterior circulation } & Anterior communicating artery & 34 & 25 & 5 & 5 & 1 \\
\hline & Pericallosal artery & 2 & 2 & & & \\
\hline & Middle cerebral artery & 3 & 18 & 1 & 6 & 1 \\
\hline & Internal carotid artery & 3 & 1 & 1 & & 1 \\
\hline & Posterior communicating artery & 17 & 8 & & 1 & \\
\hline & Anterior choroideal artery & 1 & & & & \\
\hline \multirow[t]{5}{*}{ Posterior circulation } & Basilar tip & 11 & & & & \\
\hline & Vertebral junction & 1 & & & & \\
\hline & Posterior cerebral artery & & & & & 1 \\
\hline & Posterior inferior cerebellar artery & 5 & 1 & 1 & & \\
\hline & Superior cerebellar artery & 1 & & & & \\
\hline
\end{tabular}

${ }^{a} 11$ patients had no proven aneurysm.

${ }^{\mathrm{b}}$ Five patients had false-negative CTA, in four of whom a ruptured aneurysm was diagnosed on repeat angiography. aneurysm of the middle cerebral artery was seen during surgery of a ruptured aneurysm of the anterior communicating artery. Clipping of the aneurysm of the middle cerebral artery was also performed.

\section{Inconclusive CTA result}

In 31 patients IA-DSA was performed because of inconclusive CTA results. The indications for IA-DSA examination are presented in Table 5. An overview of the results in patients in this category is presented in Fig. 1.

In 11 patients $(35 \%)$ IA-DSA confirmed the results of CTA. In 17 patients (55\%) IA-DSA was able to give further diagnostic information required for a correct patient selection for therapy. In two patients (6\%) no additional diagnostic information could was obtained from IA-DSA. In both

Table 3 Location of asymptomatic intracranial aneurysms in 224 patients

\begin{tabular}{|c|c|c|c|}
\hline \multicolumn{2}{|c|}{ Aneurysm location } & \multirow{2}{*}{$\begin{array}{l}\text { CTA- } \\
\text { positive }\end{array}$} & \multirow{2}{*}{$\begin{array}{l}\text { CTA } \\
\text { inconclusive }\end{array}$} \\
\hline $\begin{array}{l}\text { Anterior } \\
\text { circulation }\end{array}$ & $\begin{array}{l}\text { Anterior communicating } \\
\text { artery }\end{array}$ & & \\
\hline & Pericallosal artery & 2 & \\
\hline & Middle cerebral artery & $11^{\mathrm{b}}$ & 4 \\
\hline & Internal carotid artery & $9^{\mathrm{a}}$ & \\
\hline & $\begin{array}{l}\text { Posterior } \\
\text { communicating artery }\end{array}$ & $6^{\mathrm{a}}$ & \\
\hline \multirow{3}{*}{$\begin{array}{l}\text { Posterior } \\
\text { circulation }\end{array}$} & Basilar tip & 1 & \\
\hline & $\begin{array}{l}\text { Junction of vertebral } \\
\text { artery }\end{array}$ & 1 & \\
\hline & $\begin{array}{r}\text { Posterior inferior } \\
\text { cerebellar artery }\end{array}$ & 1 & \\
\hline
\end{tabular}

\footnotetext{
${ }^{\text {a }}$ One false-negative on CTA.

${ }^{\mathrm{b}}$ Two false-negatives on CTA.
} 
Table 4 Size distribution of asymptomatic intracranial aneurysms in 224 patients

\begin{tabular}{lll}
\hline Size $(\mathrm{mm})$ & CTA-positive & CTA-inconclusive \\
\hline$<5$ & $30^{\mathrm{a}}$ & 4 \\
$5-9$ & 6 & 3 \\
$10-14$ & 1 & \\
\hline
\end{tabular}

${ }^{\text {a }}$ Five false-negative on CTA.

patients vasospasm of a vertebral artery resulted in an inconclusive CTA, but also excluded selective catheterization with IA-DSA. A second CTA was negative in both patients. In one patient $(3 \%)$ treatment selection was based on a falsepositive IA-DSA. CTA was inconclusive because of amalgam artefacts in the region of the right posterior inferior cerebellar artery (PICA). An aneurysm of the right PICA was diagnosed on the first IA-DSA. A second IA-DSA was performed with the intention of coiling. However, with additional views the aneurysm turned out to be a vessel loop.

No ruptured aneurysms were found in ten patients (four nonperimesencephalic SAH, four with perimesencephalic
SAH and two negative on unenhanced CT). In 20 patients 20 ruptured intracranial aneurysms and 6 additional unruptured aneurysms were found. In one patient only an asymptomatic aneurysm was diagnosed (Tables 1, 2, 3 and 4). Of the ruptured aneurysms, 12 were clipped and 6 were coiled. Two ruptured aneurysms were clipped after failure of endovascular treatment. Two asymptomatic aneurysms were clipped.

\section{Negative CTA result}

In 60 patients CTA was considered negative. An overview of the results in this category of patients is presented in Fig. 1. Of these 60 patients, $13(22 \%)$ had negative findings on unenhanced $\mathrm{CT}$, and $47(78 \%)$ had positive findings on unenhanced CT, and of the latter 30 had a perimesencephalic blood distribution and 17 had a nonperimesencephalic SAH [16]. In $11(85 \%)$ of those with negative findings on unenhanced CT, one IA-DSA was done. In one patient a second IA-DSA was performed and in one patient a second CTA was performed. No rebleedings occurred. CTA was true-negative in all these patients.

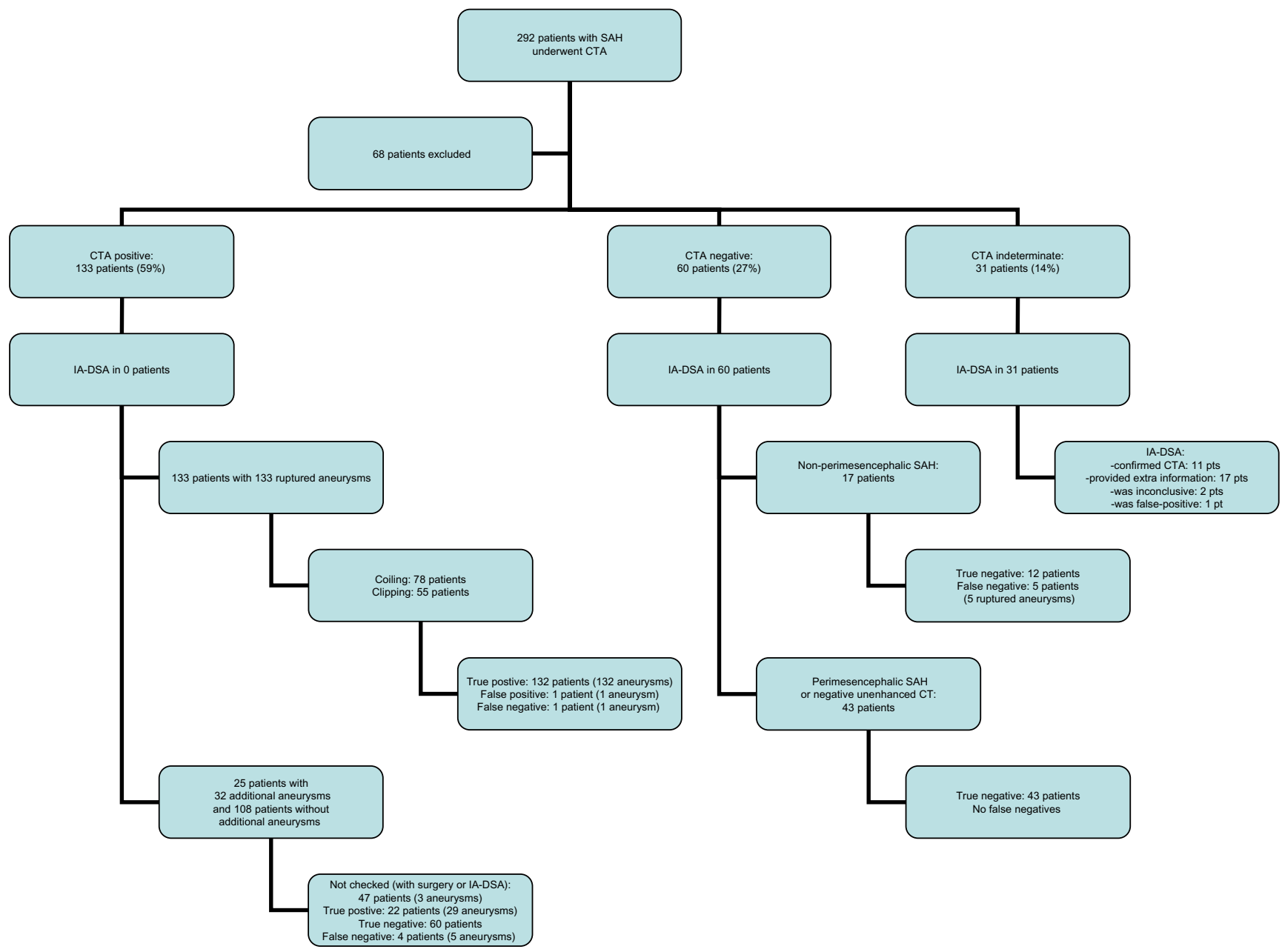

Fig. 1 Flow chart of CTA results 
Fig. 2 CTA and IA-DSA results in a 66-year-old woman with SAH Hunt and Hess grade III. CTA showed four aneurysms: an aneurysm of the anterior communicating artery (AComA) and two bilateral aneurysms of the middle cerebral artery and one aneurysm of the pericallosal artery. The aneurysm of the AComA was regarded as symptomatic at the time of initial SAH and its CTA-proven morphology showed both coiling and clipping to be a difficult challenge. Coiling of the AComA aneurysm was tried on the 2 nd day. The session was aborted because the neck of the aneurysm was broad and the aneurysm incorporated both A2 segments. Unfortunately, rebleeding occurred after coiling. The morphology of the aneurysm excluded complete occlusion by clipping on the 25 th day. In the postoperative course again two rebleedings occurred and the patient died. At autopsy a ruptured pericallosal aneurysm was seen more distal to the clipped aneurysm. a Coronal MIP CTA; b volumerendered CTA; c AP view DSA, selective catheterization of left internal carotid artery;

d volume-rendered IA-DSA;

e, $\mathbf{f}$ autopsy (red arrow anterior communicating artery aneurysm, yellow arrow middle cerebral aneurysm, black arrow anterior cerebral artery (A2 segment), blue arrow pericallosal artery aneurysm
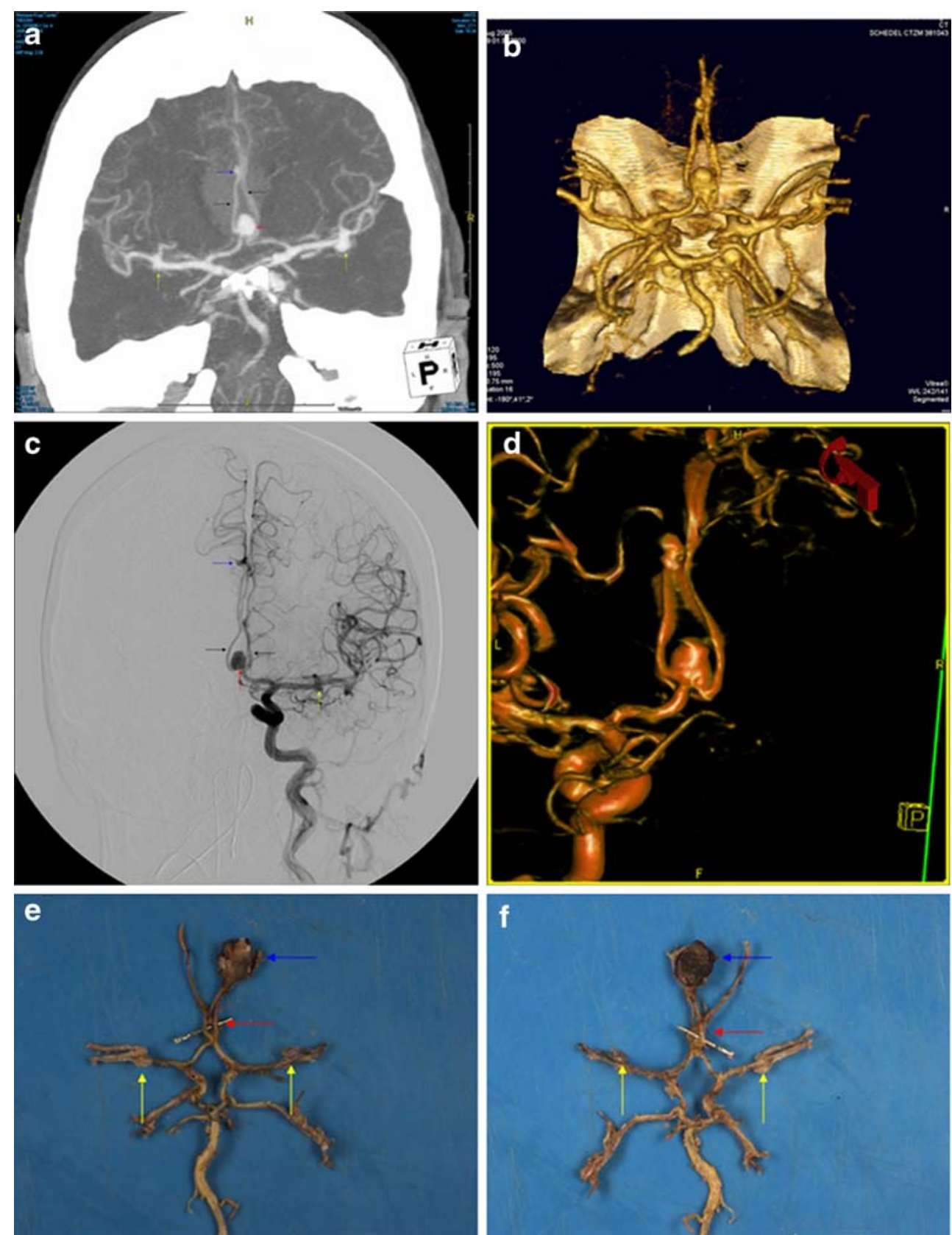

In 21 patients (70\%) with perimesencephalic SAH, IADSA was performed once, and in one of them CTA was repeated once and in one CTA was repeated twice. In eight patients IA-DSA was repeated once and in one patient IADSA was repeated twice. In this category CTA was truenegative in all these patients. No rebleedings occurred.

In nine patients $(53 \%)$ with nonperimesencephalic SAH IA-DSA was performed once, and in two of them a followup MRA was done and in one CTA was repeated. In seven patients a second IA-DSA was done. In one patient IA-DSA was repeated twice. In five patients $(29 \%)$ with nonperimesencephalic SAH, CTA was false-negative (Tables 1 and 2). In one of these patients only IA-DSA was able to detect a 3-mm ruptured aneurysm of the anterior commu- nicating artery, and in the other four a rebleeding occurred despite an initially negative CTA and IA-DSA. Repeat angiography was performed in three patients: a ruptured aneurysm was shown by CTA in two and by IA-DSA in one. One patient died before repeat angiography. Two patients were treated successfully. One patient died before treatment due to the direct effect of the rebleeding. The explanations for false-negative results were interpretation mistakes (two aneurysms recognized retrospectively on CTA and IA-DSA), and haematoma demonstrated on CTA surrounding and compressing the aneurysm leading to interpretation error on both CTA and IA-DSA in one patient and on only CTA in another (IA-DSA showed the aneurysm); the findings were uncertain in one patient. 
Table 5 Indications for IA-DSA examination in 31 patients

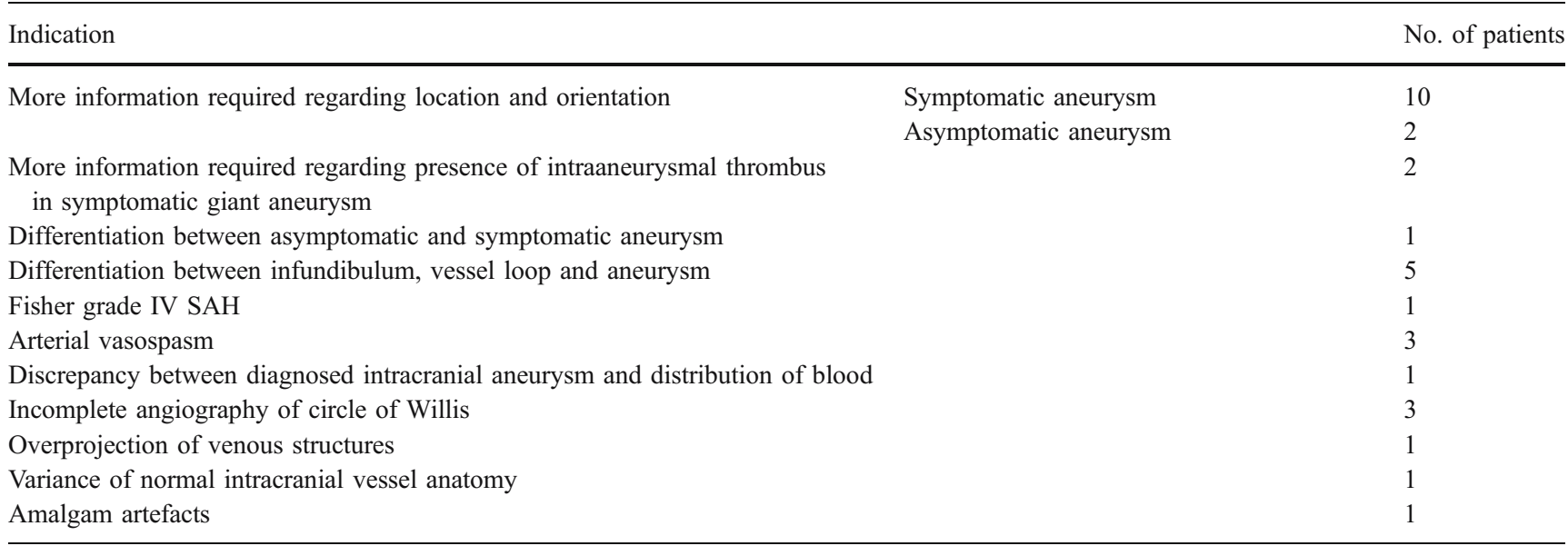

\section{Statistical analysis}

The diagnostic value of both 16- and 64-slice CTA are outlined in Tables 6 and 7. A comparison of the results of 16-and 64-slice CTA is presented in Table 8. No statistically significant differences were found.

\section{Discussion}

Our primary aim was to assess whether CTA is useful clinically in planning and performing clipping or coiling, especially in the acute phase in ruptured intracranial aneurysms, without recourse to IA-DSA. We demonstrated that it was possible to treat more than half of all patients with a ruptured intracranial aneurysm using only CTA. By avoiding conventional angiography, it was possible to streamline the management of ruptured aneurysm during the acute phase. Further, 3D-CTA was able to help in deciding whether to clip or to coil; in only two patients was treatment conversion needed due to incorrect treatment selection based on CTA.

We found 3D-CTA to be a simple, reliable, quick and minimally invasive imaging modality that reduces the risk

Table 6 Diagnostic value of CTA in ruptured aneurysms

\begin{tabular}{ll}
\hline Diagnostic value & \\
\hline True positive & 132 patients \\
False positive & 1 patient \\
True negative & 55 patients \\
False negative & 6 patients \\
Positive predictive value & $99 \%$ \\
Negative predictive value & $90 \%$ \\
Sensitivity & $96 \%$ \\
Specificity & $98 \%$ \\
Accuracy & $96 \%$ \\
\hline
\end{tabular}

of complications caused by conventional angiography and reduces the delay between the patient's arrival at the hospital and treatment, leading to diminished rebleeding. Matsumoto et al. analyzed the rate of rebleeding of ruptured aneurysms during CTA and conventional angiography, and found $0 \%$ (none of 160 patients) for CTA and 1.5\% (5 of 317 patients) for conventional angiography [17]. In patients with a ruptured aneurysm and intracerebral haemorrhage CTA saves time when aiming for a fast clot removal. Another advantage is that the radiation dosage is low compared to IA-DSA $(1.0 \mathrm{mSv}$ at $200 \mathrm{mAs}$ with the CTA Siemens Sensation 16 and $1.8 \mathrm{mSv}$ at $380 \mathrm{mAs}$ with the CTA Siemens Sensation 64 compared with 3.5-6.5 mSv with conventional angiography). Furthermore, the cost of CTA is one-fourth that of conventional angiography.

Several other studies assessing whether CTA may serve as the sole imaging method for the preoperative work-up of patients with ruptured intracranial aneurysms have been published [17-26]. An overview of these previous studies is presented in Table 9. There is a wide variation in the percentage of patients who have had their symptomatic aneurysms treated based on CTA. This may be influenced positively by the very high aneurysm prevalence and the subsequent very low negative rates of CTA in some studies $[22,23,25]$. In other studies patients with a negative CTA were not enrolled at all $[17,20]$. In general, a mean of $15-$ $20 \%$ negative angiographies after SAH is accepted [27]. The present study showed a high negative rate for CTA. This may reflect the good awareness of the diagnosis SAH in first-line and second-line health-care and the good access to CTA when the diagnosis SAH is considered. Furthermore, the wide variation in CTA-based treatment may be partially explained by differences in hardware and software used by each group, the rate of technical failures in performing CTA, scanning parameters set for screening the circle of Willis and more peripheral vessels, the experience and scrutiny of the neuroradiologist evaluating 
Table 7 Diagnostic value of CTA in additional aneurysms

Diagnostic value

Total number detected with CTA

Presence checked

True positive

False positive

True negative

False negative

Positive predictive value

Negative predictive value

Sensitivity

Specificity

Accuracy
25 patients ( 32 aneurysms)

22 patients (29 aneurysms)

22 patients (29 aneurysms)

0

120 patients $^{\mathrm{a}}$

4 patients (5 aneurysms)

$100 \%$

$97 \%$

$85 \%$

$100 \%$

$97 \%$
${ }^{a}$ Including 60 CTA-negative patients and 60 CTA-positive patients. Of the CTA-positive patients, 44 were not examined with IA-DSA as standard control.

each CTA and the willingness of the neurosurgeon and neurointerventional radiologist to rely on CTA alone in each individual case.

In the present study CTA was false-negative in $8 \%$ of patients. The risk of rebleeding after a negative initial CTA was $7 \%$. All false-negatives were in patients with a nonperimesencephalic blood distribution, giving a false- negative rate of $29 \%$ and a risk of rebleeding of $24 \%$. It seems unlikely that the false-negative rate of initial CTA and the risk of rebleeding despite a negative initial CTA in patients with a nonperimesencephalic SAH might be influenced negatively by the use of CTA as the first diagnostic tool. Firstly, in all patients with a rebleeding, repeat IA-DSA was also false-negative. Secondly, repeat angiography with CTA performed after a rebleeding still demonstrated an aneurysm.

Furthermore, the findings of other studies using IA-DSA as the first diagnostic tool were similar. In the study by Urbach et al. in 67 patients with a negative initial angiogram after SAH, four ruptured aneurysms were revealed by repeat angiography [28]. Three patients presented with a nonperimesencephalic SAH and one presented with a perimesencephalic SAH. In the study by Bradac et al., 60 of the 440 patients presenting with spontaneous SAH had a negative angiogram [29]. A second angiogram performed 1-4 weeks later revealed a ruptured aneurysm in 5 of the 40 patients. Of these patients, 3 had a second SAH. In all patients a nonperimesencephalic blood distribution was seen on CT.

Because in the present study some aneurysms could be correctly identified retrospectively, we suggest that if, under strong clinical suspicion of a ruptured aneurysm, the CTA

Table 8 Comparison of results of 16- and 64-slice CTA for detection of intracranial aneurysms

\begin{tabular}{|c|c|c|c|}
\hline & & 16 -slice ( $n=140$ patients) & 64-slice ( $n=84$ patients) \\
\hline \multirow[t]{3}{*}{ CTA result } & Positive & 74 & 59 \\
\hline & Negative & 45 & 15 \\
\hline & Inconclusive $^{a}$ & 21 & 10 \\
\hline \multirow[t]{9}{*}{ Ruptured aneurysms } & True positive & 73 & 59 \\
\hline & False positive & 1 & 0 \\
\hline & True negative & 42 & 13 \\
\hline & False negative & $4^{\mathrm{b}}$ & 2 \\
\hline & Positive predictive value (\%) & 99 & 100 \\
\hline & Negative predictive value $(\%)$ & 91 & 87 \\
\hline & Sensitivity (\%) & 95 & 97 \\
\hline & Specificity (\%) & 98 & 100 \\
\hline & Accuracy $(\%)$ & 96 & 97 \\
\hline \multirow[t]{11}{*}{ Unruptured aneurysms } & Total number on CTA & 12 (15 aneurysms) & 13 (17 aneurysms) \\
\hline & Presence checked & 11 (14 aneurysms) & 11 (15 aneurysms) \\
\hline & True positive & 11 (14 aneurysms) & 11 (15 aneurysms) \\
\hline & False positive & 0 & 0 \\
\hline & True negative & $75^{\mathrm{c}}$ & $45^{\mathrm{d}}$ \\
\hline & False negative & 1 (1 aneurysm) & 3 (4 aneurysms) \\
\hline & Positive predictive value (\%) & 100 & 100 \\
\hline & Negative predictive value (\%) & 99 & 94 \\
\hline & Sensitivity $(\%)$ & 92 & 79 \\
\hline & Specificity (\%) & 100 & 100 \\
\hline & Accuracy $(\%)$ & 99 & 95 \\
\hline
\end{tabular}

${ }^{a}$ Patients with an inconclusive result were not included in the statistical analysis.

${ }^{\mathrm{b}}$ Including one CTA-positive patient.

${ }^{\mathrm{c}}$ Including 45 CTA-negative patients and 30 CTA-positive patients. Of the CTA-positive patients, 31 were not examined with IA-DSA as standard control.

${ }^{\mathrm{d}}$ Including 15 CTA-negative patients and 30 CTA-positive patients. Of the CTA-positive patients, 13 were not examined with IA-DSA. 
Table 9 Presentation of previous studies and present study

\begin{tabular}{|c|c|c|c|c|c|c|c|}
\hline \multirow[t]{2}{*}{ Study } & \multirow{2}{*}{$\begin{array}{l}\text { No. of } \\
\text { patients }\end{array}$} & \multicolumn{3}{|c|}{ CTA-positive } & \multicolumn{2}{|c|}{ CTA-negative } & \multirow{2}{*}{$\begin{array}{l}\text { CTA inconclusive or no } \\
\text { CTA-based treatment } \\
\text { Total patients }\end{array}$} \\
\hline & & $\begin{array}{l}\text { Total } \\
\text { patients }\end{array}$ & $\begin{array}{l}\text { CTA-based } \\
\text { treatment }\end{array}$ & $\begin{array}{l}\text { True-positive } \\
\text { CTA }\end{array}$ & $\begin{array}{l}\text { Total } \\
\text { patients }\end{array}$ & $\begin{array}{l}\text { True-negative } \\
\text { CTA }\end{array}$ & \\
\hline 21 & 87 & $46(55 \%)$ & $44(96 \%)$ & $44(100 \%)$ & $15(17 \%)$ & $6(60 \%)$ & $26(30 \%)$ \\
\hline 22 & 109 & $88(81 \%)$ & 87 (99\%) & $87(100 \%)$ & $5(5 \%)$ & $5(100 \%)$ & $16(15 \%)$ \\
\hline 23 & 84 & $62(74 \%)$ & $62(100 \%)$ & $62(100 \%)$ & $7(8 \%)$ & $0(0 \%)$ & $15(18 \%)$ \\
\hline 19 & 90 & $45(100 \%)$ & $45(100 \%)$ & $45(100 \%)$ & - & - & $45(50 \%)$ \\
\hline 18 & 150 & $61(41 \%)$ & $61(100 \%)$ & $60(98 \%)^{\mathrm{a}}$ & $24(16 \%)$ & $24(100 \%)$ & $65(43 \%)$ \\
\hline 25 & 120 & $40(27 \%)$ & $40(100 \%)$ & $40(100 \%)$ & $13(11 \%)$ & $13(100 \%)$ & $67(56 \%)$ \\
\hline 24 & 78 & $27(35 \%)$ & $27(100 \%)$ & $27(100 \%)$ & $20(26 \%)$ & $20(100 \%)^{b}$ & $31(40 \%)$ \\
\hline 17 & 100 & $93(93 \%)$ & $93(100 \%)$ & $93(100 \%)$ & - & - & $7(7 \%)$ \\
\hline 20 & 96 & 87 (91\%) & $87(100 \%)$ & $86(99 \%)^{2}$ & - & - & $9(9 \%)$ \\
\hline 26 & 61 & $44(72 \%)$ & $44(100 \%)$ & $44(100 \%)$ & $15(25 \%)$ & $14(93 \%)$ & $2(3 \%)$ \\
\hline Present study & 224 & $133(59 \%)$ & $133(100 \%)$ & $132(99 \%)^{2}$ & $60(27 \%)$ & $55(92 \%)$ & $31(14 \%)$ \\
\hline
\end{tabular}

${ }^{a}$ One false-negative and one false-positive ruptured aneurysm in one patient.

${ }^{\mathrm{b}}$ In five patients with perimesencephalic SAH, IA-DSA as the gold standard control was not performed.

is reported as normal, the study should be reviewed by a second neuroradiologist before proceeding to repeat angiography. It is essential to perform a review of axial raw source images. Next, we recommend repeat CTA or IADSA when the initial CTA is negative in patients with a nonperimesencephalic SAH. There is no consensus about the time interval for repeat angiography. In practice, the guideline is to repeat angiography after several days to months. The substantial risk of rebleeding in patients with an aneurysmal pattern of haemorrhage in the present study indicates that some cerebral aneurysms are occult on initial CTA. Several factors may explain this finding. Most importantly, there is a learning curve in assessing aneurysms on CTA. Pedersen et al. reported an increase in sensitivity from $88 \%$ to $94 \%$ after 1 year's experience [30]. Small aneurysms can be missed when using CTA. CTA had a sensitivity of $50 \%$ for aneurysms $<2 \mathrm{~mm}$ in the study of Wintermark et al. [13]. Distal pericallosal and PICA aneurysms can be missed when restricting the area of coverage to the proximal circle of Willis [31-34]. Thrombosis of the neck of the aneurysm or of the entire sac is another possible reason [23]. Perianeurysmal blood or haematoma may reduce lesion conspicuity [34]. Aneurysms may be mistaken for vascular infundibula (persistent fetal nonaneurysmal dilatation of the proximal vessel) of the posterior communicating or anterior choroidal artery origins if a vessel cannot be identified arising from them [35]. Aneurysms may masquerade as tight vascular loops if the MIP thickness is wide $(>3 \mathrm{~mm})$ [34]. In patients with multiple intracranial aneurysms large aneurysms may obscure smaller ones on the CT reconstruction [33]. Aneurysms close to bone (e.g. carotid siphon, ophthalmic and posterior communicating artery) may be overlooked when relying on surface-rendering and volume-rendering techniques or using MIP with bone editing [32, 34, 36-38]. Aneurysms located within or close to the cavernous sinuses are easy to overlook unless thin-section axial and coronal MIP images are reviewed on a slightly wider window width [9].

In patients with a perimesencephalic SAH the chance of finding a posterior fossa aneurysm is low: $2.5-5 \%$ [39, 40]. Nonaneurysmal perimesencephalic haemorrhage carries no risk of vasospasm and rebleeding and has been shown to follow a benign course with an excellent prognosis [41]. The chance of finding an aneurysm in $5 \%$ of patients has to be weighed against the risk of complications from angiography imposed upon the remaining $95 \%$ of patients. CTA has a high accuracy for diagnosis of vertebrobasilar aneurysms and of intracranial aneurysms in general [9-13, 42]. In the present study, in patients with a perimesencephalic SAH and a negative initial CTA, no rebleedings occurred and CTA was true-negative in all. Similarly, in the prospective study of Huttner et al., 69 patients with a perimesencephalic SAH had a negative initial CTA and IADSA [43]. A repeat IA-DSA was performed in 38 patients $(55 \%)$. None of the repeat IA-DSAs showed any additional distinctive features with respect to the first IA-DSA. It therefore seems practical and safe to perform CTA as the first diagnostic tool and to omit repeat angiography if CTA is negative. A formal decision analysis based on these observations confirmed that a strategy where CTA is performed and not followed by conventional angiography, if negative, results in a better utility than a strategy of CTA followed by conventional angiography or of conventional angiography as primary investigation [44].

According to the results of the present study, it seems important to distinguish the two patterns of SAH on CT. 
The CT criteria of perimesencephalic bleeding have been defined [40]. Different data show that experienced radiologists can accurately discriminate between a perimesencephalic and nonperimesencephalic SAH [12, 40, 45]. Early $\mathrm{CT}$ within 3 days is necessary for reliable assessment of the pattern of haemorrhage $[12,40,46]$.

A criticism of this study might be that patients treated with endovascular coiling underwent IA-DSA as part of the endovascular procedure and thus should not be counted in the analysis of efficacy of the prospective protocol. However, a shift in management of ruptured intracranial aneurysm from surgery to endovascular treatment has appeared [47]. Endovascular treatment is replacing clipping. The use of CTA as the initial investigation for cerebral aneurysms may offset some of this increased workload whilst also improving workflow.

In conclusion, in this evaluation of the use of 16-row and 64-row multislice CTA in the management of ruptured intracranial aneurysms, we demonstrated that CTA can be used as the first-line diagnostic modality for the management of SAH patients. In CTA-negative patients IA-DSA provided no or marginal added value. IA-DSA is not needed in patients with negative CTA and classic perimesencephalic SAH. Repeat IA-DSA or CTA should still be performed in patients with a nonperimesencephalic SAH, due to false-negative CTAs and IA-DSAs in this patient group. The remaining true indication for IA-DSA was in patients with an inconclusive CTA result. In more than half of those IA-DSA provided relevant new diagnostic information.

Conflict of interest statement We declare that we have no conflict of interest.

Acknowledgement We thank W. Geuken, MD PhD, Department of Pathology, University Medical Center Groningen, The Netherlands, for supplying Fig. 2e.

\section{References}

1. Kirkpatrick PJ (2002) Subarachnoid haemorrhage and intracranial aneurysms: what neurologists need to know. J Neurol Neurosurg Psychiatry 73 (Suppl 1):i28-i33

2. Van Gijn J, Rinkel GJE (2001) Subarachnoid haemorrhage: diagnosis, causes and management. Brain 124:249-278

3. Hop JW, Rinkel GJ, Algra A, Van Gijn J (1997) Case-fatality rates and functional outcome after subarachnoid hemorrhage: a systematic review. Stroke 28:660-664

4. Cloft HJ, Joseph GJ, Dion JE (1999) Risk of cerebral angiography in patients with subarachnoid hemorrhage, cerebral aneurysm, and arteriovenous malformation: a meta-analysis. Stroke 30:317-320

5. Willinsky RA, Taylor SM, terBrugge K, Farb RI, Tomlinson G, Montanera W (2003) Neurologic complications of cerebral angiography: prospective analysis of 2,899 procedures and review of the literature. Radiology 227:522-528

6. Grzyska U, Freitag J, Zeumer H (1990) Selective cerebral intraarterial DSA. Complication rate and control of risk factors. Neuroradiology 32:296-299
7. Kuhn J, Vehlen C, Mennel HD, Mahkorn D, Bewermeyer H (2003) Rupture of an internal carotid artery aneurysm during angiography with leakage of contrast medium via an external ventricular drain. Neuroradiology 45:905-907

8. Nakatsuka M, Mizuno S, Uchida A (2002) Extravasation on threedimensional CT angiography in patients with acute subarachnoid hemorrhage and ruptured aneurysm. Neuroradiology 44:25-30

9. Dammert S, Krings T, Moller-Hartmann W, Ueffing E, Hans FJ, Willmes K, Mull M, Thron A (2004) Detection of intracranial aneurysms with multislice CT: comparison with conventional angiography. Neuroradiology 46:427-434

10. Tipper G, King-Im JM, Price SJ, Trivedi RA, Cross JJ, Higgins NJ, Farmer R, Wat J, Kirollos R, Kirkpatrick PJ, Antoun NM, Gillard JH (2005) Detection and evaluation of intracranial aneurysms with 16row multislice CT angiography. Clin Radiol 60:565-572

11. Uysal E, Yanbuloglu B, Erturk M, Kilinc BM, Basak M (2005) Spiral CT angiography in diagnosis of cerebral aneurysms of cases with acute subarachnoid hemorrhage. Diagn Interv Radiol 11:77-82

12. Velthuis BK, Rinkel GJE, Ramos LMP, Witkamp TD, van Leeuwen MS (1999) Perimesencephalic hemorrhage - exclusion of vertebrobasilar aneurysms with CT angiography. Stroke 30:1103-1109

13. Wintermark M, Uske A, Chalaron M, Regli L, Maeder P, Meuli R, Schnyder P, Binaghi S (2003) Multislice computerized tomography angiography in the evaluation of intracranial aneurysms: a comparison with intraarterial digital subtraction angiography. J Neurosurg 98:828-836

14. Kouskouras C, Charitanti A, Giavroglou C, Foroglou N, Selviaridis P, Kontopoulos V, Dimitriadis AS (2004) Intracranial aneurysms: evaluation using CTA and MRA. Correlation with DSA and intraoperative findings. Neuroradiology 46:842-850

15. Hunt WE, Hess RM (1968) Surgical risk as related to the time of intervention in the repair of intracranial aneurysms. J Neurosurg 28:14-20

16. Watanabe A, Hirano K, Kamada M, Imamura K, Ishii N, Sekihara Y, Suzuki Y, Ishii R (2002) Perimesencephalic nonaneurysmal subarachnoid haemorrhage and variations in the veins. Neuroradiology 44:319-325

17. Matsumoto M, Sato M, Nakano M, Endo Y, Watanabe Y, Sasaki T, Suzuki K, Kodama N (2001) Three-dimensional computerized tomography angiography-guided surgery of acutely ruptured cerebral aneurysms. J Neurosurg 94:718-727

18. Anderson GB, Steinke DE, Petruk KC, Ashforth R, Findlay JM (1999) Computed tomographic angiography versus digital subtraction angiography for the diagnosis and early treatment of ruptured intracranial aneurysms. Neurosurgery 45:1315-1320

19. Boet R, Poon WS, Lam JMK, Yu SCH (2003) The surgical treatment of intracranial aneurysms based on computer tomographic angiography alone - streamlining the acute management of symptomatic aneurysms. Acta Neurochir 145:101-105

20. Dehdashti AR, Rufenacht DA, Delavelle J, Reverdin A, de Tribolet N (2003) Therapeutic decision and management of aneurysmal subarachnoid haemorrhage based on computed tomographic angiography. Br J Neurosurg 17:46-53

21. Gonzalez-Darder JM, Pesudo-Martinez JV, Feliu-Tatay RA (2001) Microsurgical management of cerebral aneurysms based in CT angiography with three-dimensional reconstruction (3D-CTA) and without preoperative cerebral angiography. Acta Neurochir 143:673-679

22. Hoh BL, Cheung AC, Rabinov JD, Pryor JC, Carter BS, Ogilvy CS (2004) Results of a prospective protocol of computed tomographic angiography in place of catheter angiography as the only diagnostic and pretreatment planning study for cerebral aneurysms by a combined neurovascular team. Neurosurgery 54:1329-1340

23. Pechlivanis I, Schmieder K, Scholz M, Konig M, Heuser L, Harders A (2005) 3-Dimensional computed tomographic angiog- 
raphy for use of surgery planning in patients with intracranial aneurysms. Acta Neurochir 147:1045-1053

24. Velthuis BK, van Leeuwen MS, Witkamp TD, Ramos LMP, van der Sprenkel JMB, Rinkel GJE (1999) Computerized tomography angiography in patients with subarachnoid hemorrhage: from aneurysm detection to treatment without conventional angiography. J Neurosurg 91:761-767

25. Zouaoui A, Sahel M, Marro B, Clemenceau S, Dargent N, Bitar A, Faillot T, Capelle L, Marsault M (1997) Three-dimensional computed tomographic angiography in detection of cerebral aneurysms in acute subarachnoid hemorrhage. Neurosurgery $41: 125-130$

26. Agid R, Lee SK, Willinsky RA, Farb RI, Terbrugge KG (2006) Acute subarachnoid hemorrhage: using 64-slice multidetector CT angiography to "triage" patients' treatment. Neuroradiology 48:787-794

27. Kassell NF, Torner JC, Jane JA, Haley EC Jr, Adams HP (1990) The International Cooperative Study on the Timing of Aneurysm Surgery. Part 2: surgical results. J Neurosurg 73:37-47

28. Urbach H, Zentner J, Solymosi L (1998) The need for repeat angiography in subarachnoid haemorrhage. Neuroradiology 40:6-10

29. Bradac GB, Bergui M, Ferrio MF, Fontanella M, Stura G (1997) False-negative angiograms in subarachnoid haemorrhage due to intracranial aneurysms. Neuroradiology 39:772-776

30. Pedersen HK, Bakke SJ, Hald JK, Skalpe IO, Anke IM, Sagsveen R, Langmoen IA, Lindegaard KE, Nakstad PH (2001) CTA in patients with acute subarachnoid haemorrhage. A comparative study with selective, digital angiography and blinded, independent review. Acta Radiol 42:43-49

31. Anderson GB, Findlay JM, Steinke DE, Ashforth R (1997) Experience with computed tomographic angiography for the detection of intracranial aneurysms in the setting of acute subarachnoid hemorrhage. Neurosurgery 41:522-527

32. White PM, Teasdale EM, Wardlaw JM, Easton V (2001) Intracranial aneurysms: CT angiography and MR angiography for detection prospective blinded comparison in a large patient cohort. Radiology 219:739-749

33. Young N, Dorsch NW, Kingston RJ (1999) Pitfalls in the use of spiral CT for identification of intracranial aneurysms. Neuroradiology 41:93-99

34. Hope JK, Wilson JL, Thomson FJ (1996) Three-dimensional CT angiography in the detection and characterization of intracranial berry aneurysms. AJNR Am J Neuroradiol 17:439-445

35. Ng SH, Wong HF, Ko SF, Lee CM, Yen PS, Wai YY, Wan YL (1997) CT angiography of intracranial aneurysms: advantages and pitfalls. Eur J Radiol 25:14-19

36. Korogi Y, Takahashi M, Katada K, Ogura Y, Hasuo K, Ochi M, Utsunomiya H, Abe T, Imakita S (1999) Intracranial aneurysms: detection with three-dimensional CT angiography with volume rendering - comparison with conventional angiographic and surgical findings. Radiology 211:497-506

37. Tomandl BF, Hastreiter P, Iserhardt-Bauer S, Kostner NC, Schempershofe M, Huk WJ, Ertl T, Strauss C, Romstock J (2003) Standardized evaluation of CT angiography with remote generation of $3 \mathrm{D}$ video sequences for the detection of intracranial aneurysms. Radiographics 23:e12

38. Wilms G, Guffens M, Gryspeerdt S, Bosmans H, Maaly M, Boulanger T, Van HL, Marchal G, Baert A (1996) Spiral CT of intracranial aneurysms: correlation with digital subtraction and magnetic resonance angiography. Neuroradiology 38 (Suppl 1): S20-S25

39. Pinto AN, Ferro JM, Canhao P, Campos J (1993) How often is a perimesencephalic subarachnoid haemorrhage CT pattern caused by ruptured aneurysms? Acta Neurochir (Wien) 124:79-81

40. Rinkel GJ, Wijdicks EF, Vermeulen M, Ramos LM, Tanghe HL, Hasan D, Meiners LC, Van Gijn J (1991) Nonaneurysmal perimesencephalic subarachnoid hemorrhage: $\mathrm{CT}$ and MR patterns that differ from aneurysmal rupture. AJNR Am J Neuroradiol 12:829-834

41. Rinkel GJ, Wijdicks EF, Vermeulen M, Hasan D, Brouwers PJ, Van Gijn J (1991) The clinical course of perimesencephalic nonaneurysmal subarachnoid hemorrhage. Ann Neurol 29:463-468

42. Karamessini MT, Kagadis GC, Petsas T, Karnabatidis D, Konstantinou D, Sakellaropoulos GC, Nikiforidis GC, Siablis D (2004) CT angiography with three-dimensional techniques for the early diagnosis of intracranial aneurysms. Comparison with intraarterial DSA and the surgical findings. Eur J Radiol 49:212-223

43. Huttner HB, Hartmann M, Kohrmann M, Neher M, Stippich C, Hahnel S, Kress B (2006) Repeated digital subtraction angiography after perimesencephalic subarachnoid hemorrhage? J Neuroradiol 33:87-89

44. Ruigrok YM, Rinkel GJ, Buskens E, Velthuis BK, Van Gijn J (2000) Perimesencephalic hemorrhage and CT angiography: a decision analysis. Stroke 31:2976-2983

45. Kallmes DF, Clark HP, Dix JE, Cloft HJ, Evans AJ, Dion JE, Kassell NF (1996) Ruptured vertebrobasilar aneurysms: frequency of the nonaneurysmal perimesencephalic pattern of hemorrhage on CT scans. Radiology 201:657-660

46. Adams HP Jr, Kassell NF, Torner JC, Sahs AL (1983) CT and clinical correlations in recent aneurysmal subarachnoid hemorrhage: a preliminary report of the Cooperative Aneurysm Study. Neurology 33:981-988

47. Molyneux A, Kerr R, Stratton I, Sandercock P, Clarke M, Shrimpton J, Holman R (2002) International Subarachnoid Aneurysm Trial (ISAT) of neurosurgical clipping versus endovascular coiling in 2143 patients with ruptured intracranial aneurysms: a randomised trial. Lancet 360:1267-1274 